\title{
A ductile fracture criterion with Zener-Hollomon parameter of pure molybdenum sheet in thermal forming
}

\author{
Chu Wang ${ }^{\mathrm{a}}$, Min Wan, Yingke Zhou, and Xiangdong Wu \\ Beihang University, School of Mechanical Engineering and Automation, 100191 Beijing, PR China
}

\begin{abstract}
Formability of pure molybdenum in thermal forming process has been greatly improved, but it is still hard to avoid the generation of rupture and other quality defects. In this paper, a ductile fracture criterion of pure molybdenum sheet in thermal forming was established by considering the plastic deformation capacity of material and stress states, which can be used to describe fracture behaviour and critical rupture prediction of pure molybdenum sheet during hot forming process. Based on the isothermal uniaxial tensile tests which performed at 993 to $1143 \mathrm{~K}$ with strain rate range from 0.0005 to $0.2 \mathrm{~s}^{-1}$, the material parameters are calculated by the combination method of experiment with FEsimulation. Based on the observation, new fracture criteria can be expressed as a function of Zener-Hollomon parameter. The critical fracture value that calculated by OyaneSato criterion increases with increasing temperature and decreasing strain rate. The ductile fracture criterion with Zener-Hollomon parameter of pure molybdenum in thermal forming is proposed.
\end{abstract}

\section{Introduction}

Molybdenum is a refractory metal which is widely utilized in lighting-technology, high performance electronics, furnace construction and sputter targets since it possesses a combination of physical properties including excellent strength at elevated temperature, high corrosion resistance except oxidation resistance, high thermal conductivity, a high elastic modulus and a low thermal-expansion coefficient. However, it is difficult to work in hot forming processes due to extensive surface and edge cracking. Therefore, it is necessary to study the fracture behaviour of pure molybdenum and establish a reasonable fracture criterion to predict the crack initiation in the design stage of the process [1].

In the past, a number of ductile fracture criteria are proposed by the quantitative description of the fracture behaviours and can be applied to the industrial environment. The application of the ductile fracture criteria for the prediction of the forming limit diagram (FLD) of sheet metal was investigated by Ozturk [2] et al. Lou [3] et al. developed a ductile fracture criterion which is proposed to model fracture behaviour of sheet metals for nucleation, growth and shear coalescence of voids during plastic deformation. The combination of FE simulation with tension tests is adopted to predict accurately the forming limit in sheet metal forming by Liu [4] et al.

\footnotetext{
${ }^{a}$ Corresponding author: wangchu711@126.com
}

This is an Open Access article distributed under the terms of the Creative Commons Attribution License 4.0, which permits unrestricted use, distribution, and reproduction in any medium, provided the original work is properly cited. 


\section{MATEC Web of Conferences}

Table 1. Chemical composition of pure molybdenum sheet (wt \%).

\begin{tabular}{|c|c|c|c|c|c|c|c|c|c|c|}
\hline $\mathbf{A l}$ & $\mathbf{O}$ & $\mathbf{S i}$ & $\mathbf{C a}$ & $\mathbf{K}$ & $\mathbf{N a}$ & $\mathbf{N i}$ & $\mathbf{W}$ & $\mathbf{F e}$ & $\mathbf{M g}$ & $\mathbf{M o}$ \\
\hline 0.005 & 0.007 & 0.005 & 0.007 & 0.005 & 0.005 & 0.005 & 0.005 & 0.007 & 0.003 & Bal. \\
\hline
\end{tabular}

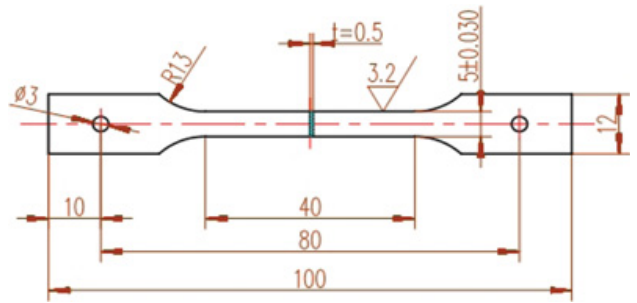

Figure 1. Geometry of molybdenum sheet specimen for hot tensile test.

The criteria mentioned above have been successfully utilized in cold metal forming processes. However, the extension of the fracture criteria to hot forming processes is difficult because ductile fracture is a complicated phenomenon that is dependent upon process parameters, such as stress, strain and particularly forming temperature. $\mathrm{He}$ [5] et al, built a new ductile fracture criterion taking into account the plastic deformation capacity of material and stress states, which can be used to describe fracture behaviour and critical rupture conditions of $30 \mathrm{Cr} 2 \mathrm{Ni} 4 \mathrm{MoV}$ ultra-super-critical rotor steel during hot forging process. Therefore, for hot forming processes, a reliable fracture criterion should at least account for temperature and strain rate.

\section{Experiments}

A commercial Mo1 pure molybdenum sheet with purities of $99.95 \%$ in fully recrystallized condition was produced by cold-isotactic pressing of molybdenum power followed by a sintering process at $2273 \mathrm{~K}$ in hydrogen furnace, and the chemical composition of test material is listed in Table 1 . Then the sintered plate was hot-rolled to a thickness of approximately $2 \mathrm{~mm}$ through cross rolling at temperature of $1573 \mathrm{~K}$ and was annealed at $1403 \mathrm{~K}$. The sheet was subsequently rolled at room temperature to a reduction of $50 \%$ and annealed at $1403 \mathrm{~K}$ for $20 \mathrm{~min}$. The cold-rolling and annealing were repeated until a thickness of $0.5 \mathrm{~mm}$ was achieved, and the sheet was finished fully recrystallized.

Specimens for hot tensile test were extracted along the rolled direction of the plates. The sample geometry is shown in Fig. 1. The hot tensile tests were carried out on Gleeble-1500 thermo-simulation machine under four different deformation temperatures (993, 1043, 1093 and $1143 \mathrm{~K}$ ) and five different strain rates $\left(0.0005,0.001,0.005,0.1\right.$ and $\left.0.2 \mathrm{~s}^{-1}\right)$ in vacuum environment [6]. First, the specimens were heated to the target deformation temperature at a heating rate of $10 \mathrm{~K} / \mathrm{min}$ and held isothermally for $3 \mathrm{~min}$ to eliminate the thermal gradient. Then, the specimens were stretched under the constant temperatures and strain rates until fracture and the true stress-strain curves are shown in Fig. 2.

\section{Fracture criterion}

In metal forming processes, the accumulation of damage usually contributes to fracture, which depends on the stress-strain path within a local region. The damage represents voids nucleation growth and coalescence into micro-cracks. In developing fracture criterion, total damage is the accumulation of damages in each time step, which is calculated according to the temperatures and strain rates. 

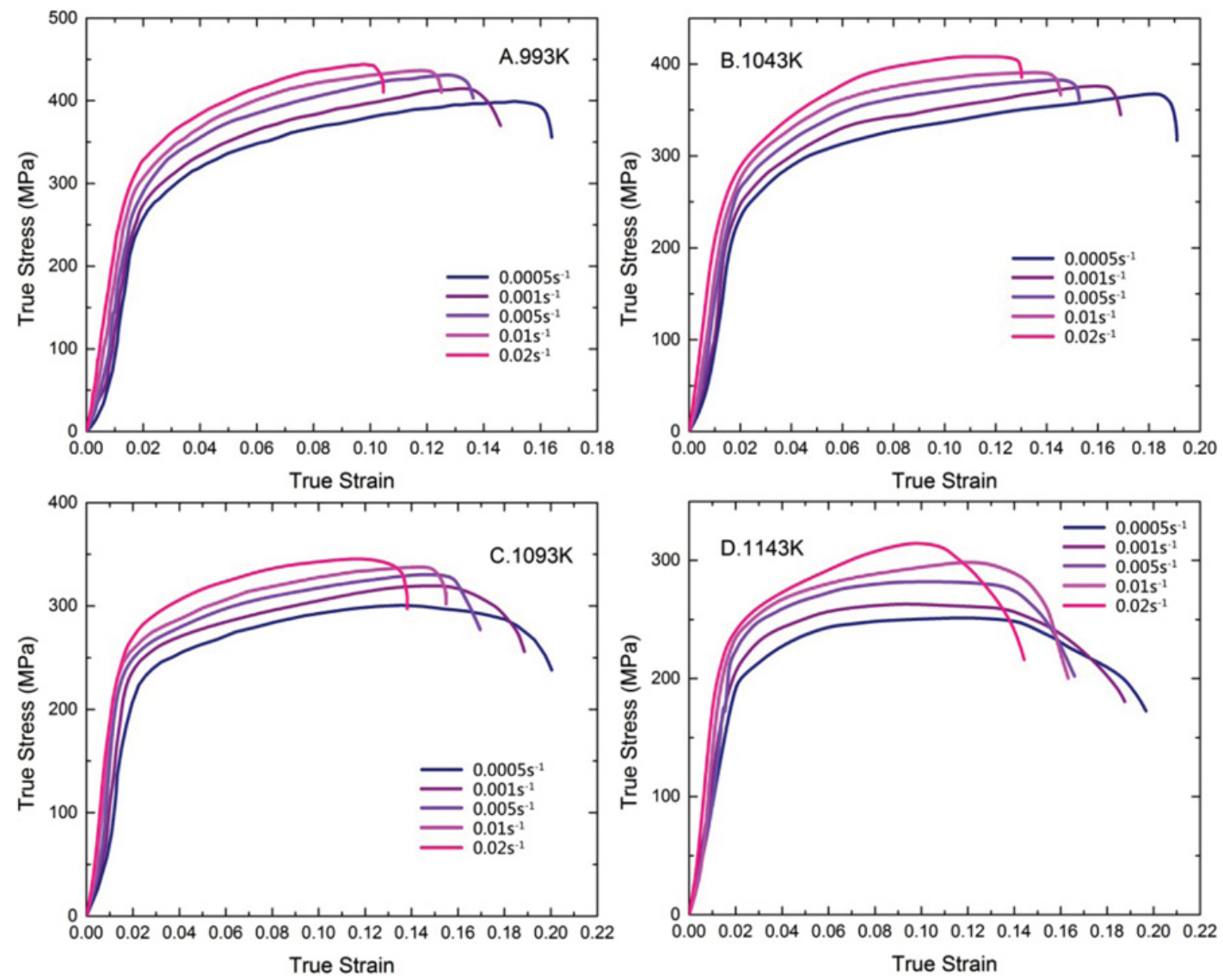

Figure 2. True stress-strain curves for pure molybdenum under temperatures: (A) $993 \mathrm{~K}$, (B) $1043 \mathrm{~K}$, (C) $1093 \mathrm{~K}$ and (D) $1143 \mathrm{~K}$.

\subsection{Critical fracture strain}

The fracture critical strain $\varepsilon_{f}$ which is defined as the equivalent strain when the necking occurs during tensile process and an important mechanical parameter, reflecting the plastic deformation capacity, is plotted as a function of temperature and strain rate in Fig. 3(a). This equivalent strain can be calculated as $\varepsilon_{f}=\ln \left(l_{f} / l_{0}\right)$ where $l_{0}$ is the initial length of the specimen and $l_{f}$ is its length at separation. It can be observed that the critical fracture strain increases with increasing temperature and decreasing strain rate. At the nominal axial strain rates of 0.01 and $0.02 \mathrm{~s}^{-1}$, all the critical fracture strains are beyond $17 \%$ and a higher strain is indicated at higher temperature. The critical fracture strain is about $21 \%$ at $1143 \mathrm{~K}$ and $0.0005 \mathrm{~s}^{-1}$. Thus, $\varepsilon_{f}$ reatly depends on the temperature and strain rate in hot forming. The effects of temperature and strain rate on deformation behavior can be expressed with Zener-Hollomon parameter $Z$ in an exponent-type equation

$$
Z=\dot{\varepsilon} \exp \left(\frac{Q}{R T}\right)
$$

where $\dot{\varepsilon} \mathrm{s}$ the strain rate, $\mathrm{s}^{-1} ; Q$ is the apparent activation energy, $\mathrm{J} \cdot \mathrm{mol}^{-1} R$ is the universal gas constant, $8.314 \mathrm{~J} \cdot \mathrm{mol}^{-1} \cdot \mathrm{K} ; T$ is the thermodynamics temperature. Take nature logarithm on both 


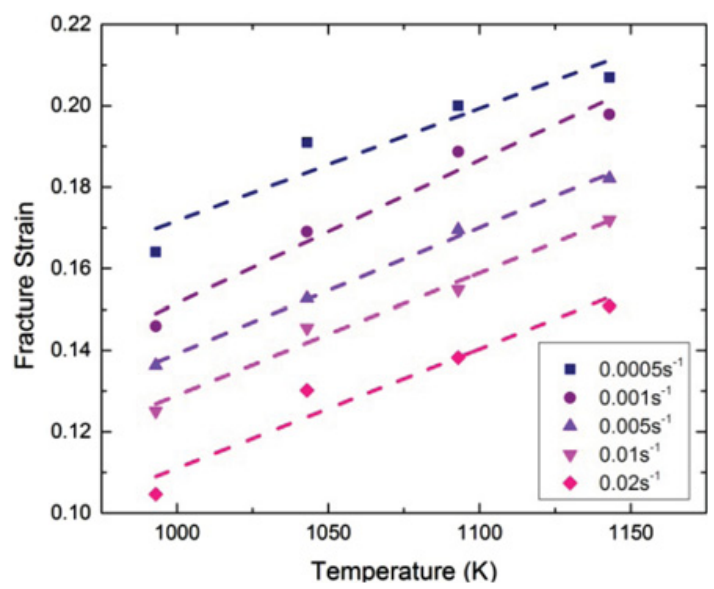

(a)

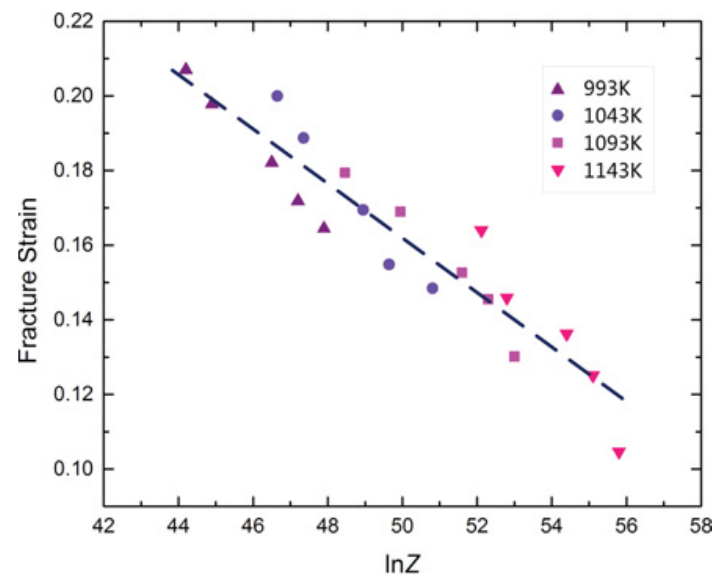

(b)

Figure 3. (a) Fracture strain as function of temperature and strain rate; (b) fracture strain as function of $\ln Z$ and temperature.

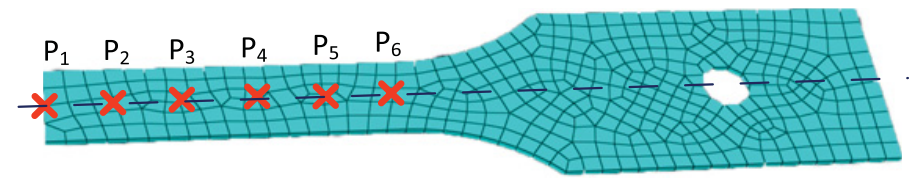

Figure 4. Selected points distribution on tensile test sample.

sides of Eq. (1), gives

$$
\ln Z=\ln \dot{\varepsilon}+\frac{Q}{R T} .
$$

Since Q/R is known for each alloy, using Fig.3(a) and Eq.(1), the fracture strain can be plotted against $\ln Z$ as Fig. 3(b). The plots appear in a linear relationship and the regression equation is $\varepsilon_{f}=0.53201-$ $0.00742 \ln Z$, which implying that fracture is mainly controlled by $\ln Z$ and this observation can be utilized in the computational analysis. Subsequently, the number of experiments that need for developing a fracture criterion may be reduced if this linear relationship is verified for other stress states. Such verification have been carried out using tension and torsion tests data on Ti alloy and Al alloy in hot forming [7].

The phenomenon that fracture strain is sensitive to $\ln Z$ is significant for both the expression of experimental results and their application to simulate metal forming processes. However, the critical fracture strain and the relationship between $\varepsilon_{f}$ and $\ln Z$ obtained by upset tests cannot be used in other stress states directly [8].

\subsection{Oyane-Sato criterion in thermal forming}

Because of the various principles and applications of fracture criteria, it is necessary to compare their validity and make a reasonable choice. In this work, the Oyane-Sato fracture criterion was chosen for the damage modeling at elevated temperatures since it accounts for the triaxiality dependency and has 


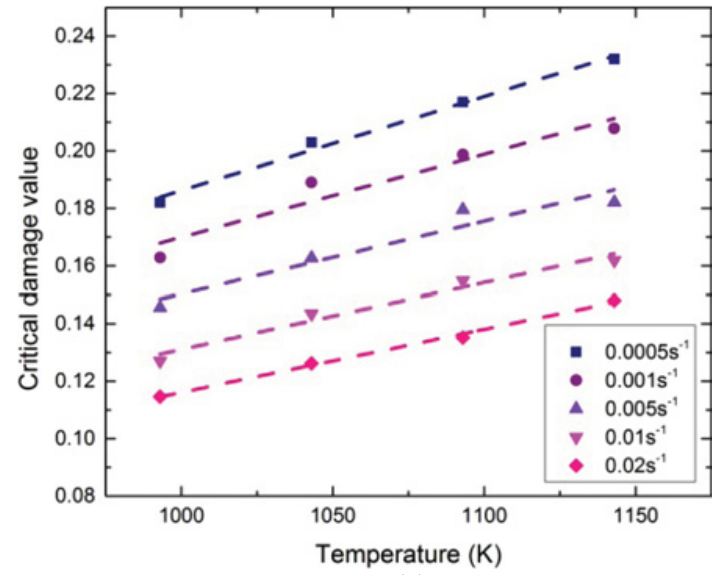

(a)

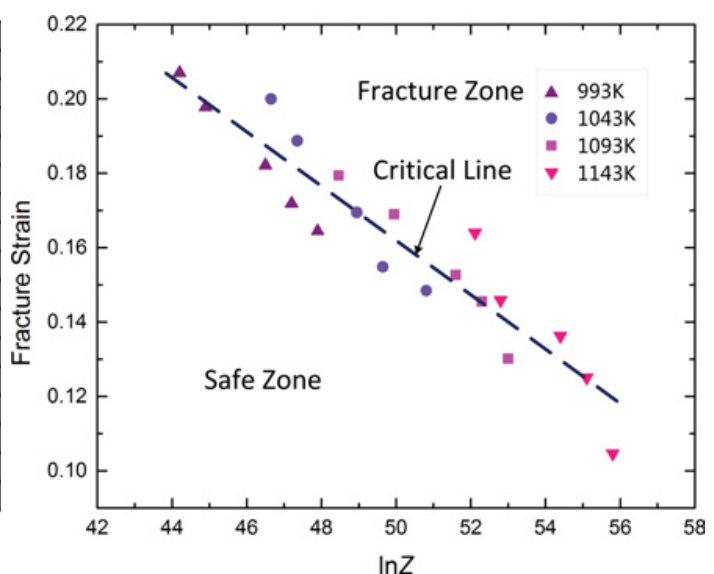

(b)

Figure 5. (a) Critical damage value as function of temperature and strain rate; (b) critical damage value as function of $\ln Z$ and temperature.

proved to be effective for medium to high triaxiality values. The conventional Oyane-Sato fracture criterion is presented as

$$
\int_{0}^{\bar{\varepsilon} f}\left(1+B \frac{\sigma_{H}}{\bar{\sigma}}\right) d \bar{\varepsilon}=C
$$

where $\sigma_{H}$ is the hydrostatic stress; $\sigma$ is the equivalent stress; $\bar{\varepsilon}$ is the effective strain; the value of coefficient $B$ is chosen equal to 3 according to literature (Oyane et al., 1980). The variation of $\sigma_{H}$ and $\sigma$ wiith the effective strain $\bar{\varepsilon}$ are obtained by FEM simulation using ABAQUS/Standard [9].

The local values of the stress and strain components during the whole history of the deformation process can be computed from the numerical simulation. The damage value $C$ of any point on the specimen can be calculated using Eq. (3) and the point with the maximum value is usually the fracture initiation site. In order to confirm the fracture initiation sites predicted by Oyane-Sato criterion, the evaluation of the Oyane integral at various locations on the equatorial plane was performed using ABAQUS/Standard simulations. The fracture initiation sites on the free surface can be successfully predicted by the Oyane-Sato fracture criterion which is in close agreement with the experimental observations. The damage value obtained by Oyane-Sato criterion in cold metal forming is usually a constant. However, in metal hot forming processes, the critical fracture values increase with increasing temperature and decreasing strain rate, showing a strong dependence on tensile test conditions, as shown in Fig. 5(a). The prediction of surface cracking of pure molybdenum by critical value $C_{f}$ is difficult because the critical fracture value is a complex function of temperature and strain rate. Therefore, it is necessary to establish a criterion that involves temperature and strain rate at least and could easily predict the crack initiation for hot forming processes.

\subsection{Ductile fracture criterion based on Zener-Hollomon parameter}

The effects of deforming temperature and strain rate on critical fracture value can also be replaced with $\ln Z$ which is similar to the analysis aforementioned. It can be observed that a linear relationship is obeyed between $C_{f}$ and $\ln Z$ from Fig. 5(b), and its regression equation gives

$$
C_{f}=0.55656-0.00766 \ln Z \text {. }
$$




\section{MATEC Web of Conferences}

This regression line is the symbol of the boundary between the fracture zone and the safe zone. If the damage value, obtained by FEM simulation under arbitrary deformation conditions, drops into the safe zone in the lower left quadrant, a sound product without failure will be obtained.

Above all, the Oyane-Sato fracture criterion for pure molybdenum at elevated temperature can be given as

$$
\begin{gathered}
C>C_{f} \\
\int_{0}^{\bar{\varepsilon} f}\left(1+3 \frac{\sigma H}{\bar{\sigma}}\right) d \bar{\varepsilon}>0.55656-0.0076 \ln Z .
\end{gathered}
$$

Equation (6) is the fracture criterion of pure molybdenum in thermal forming. The integration of the left-hand side is the damage that the material suffers during the plastic deformation, and the right-hand side is the critical fracture value $C_{f}$. This criterion implies that ductile fracture is dependent not only on the stresses and the strains imposed, but also on the combined effect of temperature and strain rate in hot forming.

It is well known that ductile fracture in hot forming depends on the local conditions of stress, strain, strain rate and temperature as well as processing methods, such as compression, tension, torsion, and extrusion. However, these processes can be expressed as a function of stress and strain that are the thermodynamics parameters. Therefore, the critical fracture value $C_{f}$ obtained by hot tensile tests, can be used to predict the facture initiation during other complex processes comparatively by FEM simulation. The introduction of this strategy may significantly reduce the number of experimental tests that have to be done in order to calibrate the critical values of workability models. However, this calculation is on the assumption that $C_{f}$ is independent on the stress states. He [5] et al. conducted cylindrical upsetting (CUP), tapered upsetting (TUP) and double-cone upsetting (DUP) with the same ratio of height and diameter $(\mathrm{H} / \mathrm{D}=1.5)$ on a $30 \mathrm{Cr} 2 \mathrm{Ni} 4 \mathrm{MoV}$ ultra super critical rotor steel at elevated temperatures. It was found that there are good agreement between the critical value and experimental results, indicating the independence of the critical fracture values on stress states. However, further experiments are required to confirm the independence between critical fracture value and stress state in thermal forming.

\section{Conclusion}

In this paper, it is found that the fracture strain and damage values evaluated from the series of uniaxial tensile tests conducted in wide ranges of strain rate and temperature could be well correlated with Zener-Hollomon parameter. Based on the observation, new fracture criteria can be expressed as a function of Zener-Hollomon parameter $\ln Z$. The critical fracture value $C_{f}$ calculated by Oyane-Sato criterion increases with increasing temperature and decreasing strain rate. $C_{f}$ and $\ln Z$ also exhibit a linear relationship. Eventually, the fracture criterion of pure molybdenum in thermal forming is

$$
\int_{0}^{\bar{\varepsilon} f}\left(1+3 \frac{\sigma H}{\bar{\sigma}}\right) d \varepsilon>0.55656-0.00766 \ln Z .
$$

This research is supported by the European Regional Development Fund and the Slovak state budget for the project "Research Centre of University of Žilina”, ITMS 26220220183. 


\section{ICNFT 2015}

\section{References}

[1] S. Primig, H. Leitner, H. Clemens, A. Lorich, W.Knabl, R. Stickler. Int. J. Refract. Met. Hard Mater 28, 703 (2010)

[2] F. Ozturk, D. Lee. J. Mater. Process. Technol. 147, 397 (2004)

[3] Y.S. Lou, H. Huh, S.J. Lim, K. Pack. Int. J. Solids. Struct. 49, 3605 (2013)

[4] H.S. Liu, Y.Y. Yang, Z.Q. Yu, Z.Z. Sun, Y.Z. Wang. J. Mater. Process. Technol. 209, 5443 (2009)

[5] J.L. He, Z.S. Cui, F. Chen, Y.H. Xiao, L.Q. Ruan. Mate. Des. 52, 547 (2013)

[6] B. Meng, M. Wan, X.D. Wu, Y.K. Zhou, C. Chang. Int. J. Refract. Met. Hard Mater. 45, 41 (2014)

[7] X.M. Zhang, W.D. Zeng, Y. Shu, Y.G. Zhou, Y.Q. Zhao, H. Wu, H.Q. Yu. Tran. Nonferrous Met. Soc. China. 19, 267 (2009)

[8] S. Alexandrov, P.T. Wang, R.E. Roadman. J. Mater. Process. Technol. 160, 257 (2005)

[9] M. Zhan, C.G. Gu, Z.Q. Jiang, L.J. Hu, H. Yang. Comput. Mater. Sci. 47, 353 (2009) 